Reprod. Nutr. Dévelop., 1984, 24 (5A), 515-527.

\title{
Stockage et mobilisation de la tyrosine chez une larve de lépi- doptère :dosages de la tyrosine, de son glucoside et d'autres acides aminés chez Spodoptera littoralis - lépidoptère : noctuidae
}

\author{
Y. RAHBÉ, B. DELOBEL, G. BONNOT
}

avec la collaboration technique de C. OGIER et J. GUILLAUD

Laboratoire de Biologie 406, I.N.S.A., I.N.R.A. 20, av. A.-Einstein, 69621 Villeurbanne, Cedex, France

Summary. Storage and mobilization of tyrosine in a lepidopterous larva : assay of tyrosine, its glucoside and other amino acids in Spodoptera littoralis, a lepidopterous noctuid moth.

An HPLC method has been developed for simultaneous determination of tyrosine and its 0-glucoside. Concentrations of both in whole tissues and in the haemolymph of a noctuid moth, Spodoptera littoralis, were determined through larval life. The presence of glucosides was assessed from the last larval instar on, the major site of storage being the haemolymph. In this sixth instar, the metabolism of tyrosine and its glucoside seemed to be thoroughly coupled. A rapid increase of $\beta$-D-glucopyranosyl-0-L-tyrosine concentration was followed by a two-step fall at the end of the instar; a moderate one soon after cessation of feeding and a sharp fall at pupal ecdysis due to the utilization of tyrosine for cuticle formation. Emphasis has been placed on the last larval instar and tyrosine level has been compared with the levels of other free amino-acids and peptides grouped according to their profile in the instar.

\section{Introduction.}

La tyrosine, acide aminé semi-indispensable chez les insectes (groupe aromatique indispensable : tyrosine et phénylalanine), est un précurseur clé d'un certain nombre de voies métaboliques, outre la synthèse protéique : catécholamines neurotransmettrices, voies de la mélanisation et de la sclérotisation. Cette dernière est une voie cruciale à plusieurs étapes de la métamorphose des insectes et l'activation de ses systèmes enzymatiques spécifiques, nécessitant alors une grande disponibilité en tyrosine, aboutit à la formation de quinones tannantes cuticulaires (Karlson et Sekeris, 1962 ; Andersen, 1971 ; Sugumaran et Lipke, 1982). Pour faire face à une demande massive lors de la mue nymphale, les lépidoptères font appel à un stockage protéique adéquat (S. J. Kramer et al., 1980 ; Telfer et al., 1983), mais surtout à une forme de petit poids moléculaire. Le $\beta$-D- 
glucopyranosyl-O-L-tyrosine ('), identifié chez Mancuda sexta (K. J. Kramer et al., 1980), doit être un moyen répandu de stockage chez les larves de lépidoptères (Ishizaki et Umebachi, 1980 ; Lu et al., 1982), même si d'autres formes semblent compléter le pool de tyrosine larvaire : tyrosine libre ou conjugué fragile pour les mues larvo-larvaires (McDermid et Locke, 1983), tyrosine peptidique et protéique. Notre travail a cherché à préciser la dynamique d'accumulation et d'utilisation de $\beta$ GIcTYR chez la noctuelle $S$. littoralis, afin d'accéder à son déterminisme hormonal et de quantifier la participation de ce composé à la voie de sclérotisation lors de la formation de la chrysalide. L'évolution des autres acides aminés a été examinée au cours du dernier stade larvaire pour dégager les particularités de l'évolution de la tyrosine non protéique.

\section{Matériel et méthodes.}

La souche de $S$. littoralis provient de la station INRA de Zoologie d'Avignon.

Animaux : Les larves sont élevées sur milieu artificiel à $25 \pm 1^{\circ} \mathrm{C}, 75 \mathrm{HR}$ et photopériode 12/12 (Poitout et Bues, 1972). Les mues sont suivies à $\pm 3 \mathrm{~h}$ sur élevage individuel.

Extraction et purification : le glucoside de la tyrosine est préparé à partir de larves de stade 6 en fin d'alimentation, par extraction aqueuse, dialyse, chromatographie sur résine échangeuse de cations (Dowex $50 W \times 4$, forme $H+$ ), et séparation finale sur colonne préparative de silice greffée $\mathrm{NH} 2130 \mathrm{~cm} ; \varnothing$ intérieur : $10 \mathrm{~mm} ; \varnothing$ des particules : $25-40 \mu \mathrm{m})$. L'élution se fait à $1 \mathrm{ml} / \mathrm{min}$. par un gradient passant en une heure de la solution $\mathrm{B}$ à un mélange à $50 \%$ de $\mathrm{A}$ et de $\mathrm{B}$ (voir plus loin).

Extraction individuelle: Pools de larves (stades 1 à 4) ou larves individuelles (stades 5 ou 6) sont broyés in toto a $0^{\circ} \mathrm{C}$ en présence de $1,5 \mathrm{ml}$ de TCA (concentration finale $3 \%$ ) et d'une quantité déterminée de standard interne (pNH2 PHE). Trois extractions par deux volumes de chloroforme, suivies de centrifugation à $4{ }^{\circ} \mathrm{C}(350 \mathrm{~g}, 10 \mathrm{~min}$.) permettent de débarrasser la phase aqueuse de lipides, des protéines précipitées et d'une partie du TCA. L'extrait conservé à $-20^{\circ} \mathrm{C}$ est amené à $\mathrm{pH}$ 6-7 par $\mathrm{NaHCO}_{3}$ en poudre préalablement à l'injection HPLC. La neutralisation est vérifiée au papier $\mathrm{pH}$ pour s'assurer d'une bonne reproductibilité des temps de rétention des différents composés, notamment de l'acide urique (fig. 1d). Aux stades 5 et 6 , l'hémolymphe des larves individuelles (10 à $150 \mu l$ ) est traitée de façon analogue, les volumes étant réduits en conséquence).

(1) Les abréviations utilisées sont, outre celles couramment admises pour les acides aminés usuels : $\beta$ glc TYR pour le $\beta$-D-glucopyranosyl-O-L-tyrosine; $\mathrm{pNH} 2$ PHE pour la $\mathrm{p}$-aminophenylalanine ; A. Am. B. (resp. B. Am. I. B.) pour l'acide $(\alpha) \beta$ amino(iso)butyrique ; G.A.B.A. pour l'acide $\gamma$ aminobutyrique; TCA pour l'acide trichloracétique; HPLC pour la chromatographie liquide sous haute pression. 

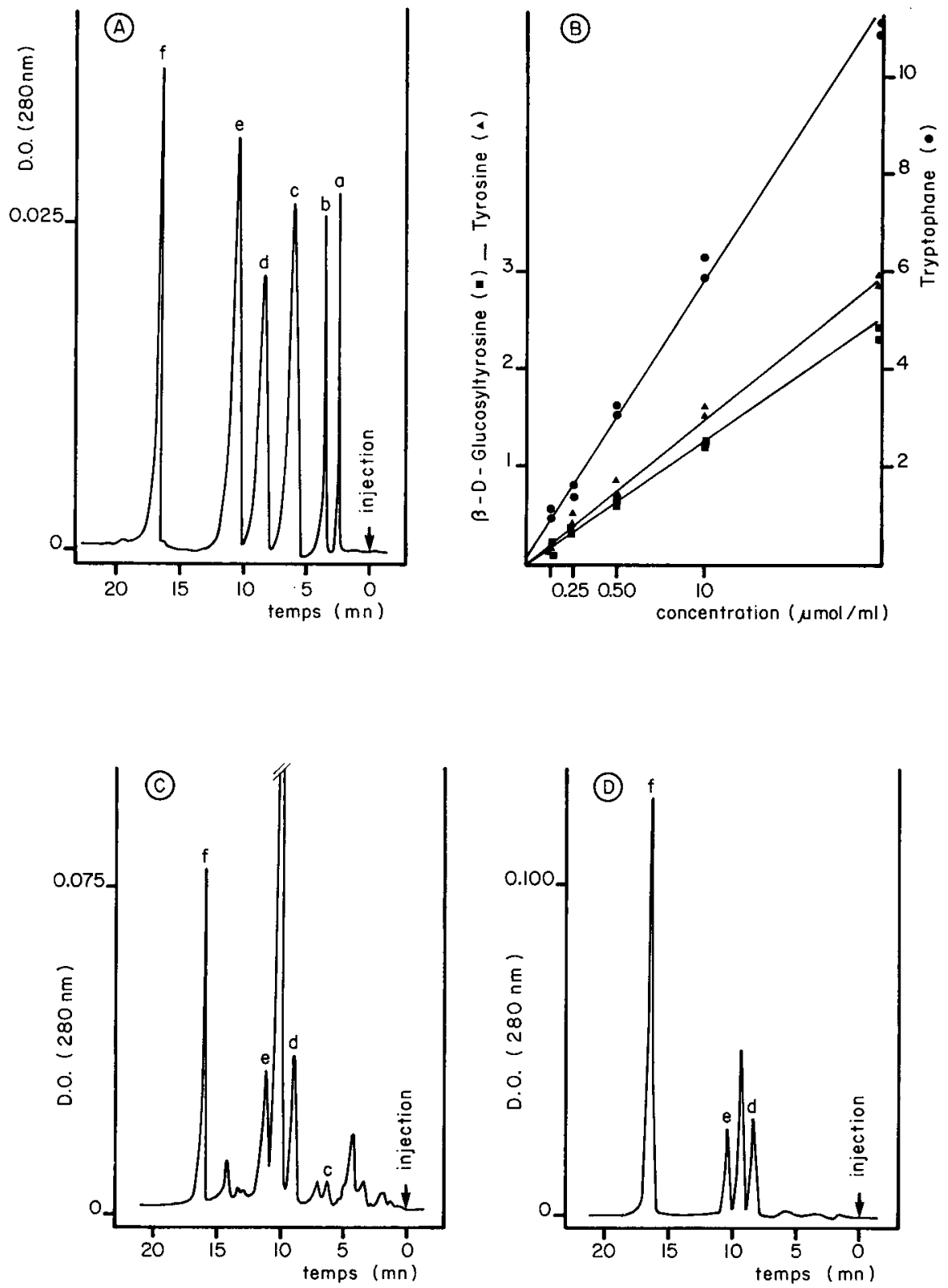

FIG. 1. - Dosage simultané de TYR et $\beta$ GIC TYR par HPLC (conditions : voir texte).

A. Chromatogramme standard: $a=\mathrm{pOH}$ phenylpropionate; $b=\mathrm{pOH}$ phenylacetate ; $c=$ tryptophane; $d=\mathrm{pNH}_{2}$ phenylalanine (Std interne) ; $\mathrm{e}=$ tyrosine ; $\mathrm{f}=\beta \mathrm{Glc}$ TYR.

B. Courbe dose/réponse du dosage (TCA + délipidation + HPLC) (voir texte).

C. Chromatogramme type d'une larve entière de stade 6 en fin d'alimentation (temps $" \mathrm{C} m$ ) ; entre les pics de p $\mathrm{NH}_{2}$ PHE (d) et TYR (e) : I'acide urique.

D. Chromatogramme type d'hémolymphe d'une larve de stade 6 (temps « $\mathrm{c}$ »). 
HPLC : colonne - silice greffée $\mathrm{NH} 2,25 \mathrm{~cm}$ de longueur, $4,6 \mathrm{~mm}$ de diamètre, particules de $5 \mu \mathrm{m}$ (Merck Lichrosob NH2).

détection - absorption U.V. à $280 \mathrm{~nm}$;

débit $-1,5 \mathrm{ml} / \mathrm{min}$;

élution - $\mathrm{A}$ : NaH2PO4 0.01M ajusté à pH 4.3; B : acétonitrile-eau (500:70), - temps $0: 97 \%$ de B dans A + B ;

- temps $0: 1,5$ à 8,5 min : gradient linéaire de $97 \%$ à $90 \%$ de $B / A+B$;

- temps 8,5 à 18,5 min : gradient linéaire de $90 \%$ à $50 \%$ de $B / A+B$;

- $18,5 \min : 30 \%$ de $B / A+B$;

$-23,5$ min : régénération en 5 min jusqu'à $97 \%$ de $B / A+B$.

La méthode est adaptée d'une séparation d'acides aminés par HPLC (Schuster, 1980). Un chromatogramme standard est donné en figure 1a (séparation d'acides et acides aminés naturels; la pNH2 PHE est utilisée comme standard interne). Les caractères de linéarité du dosage total sur les hauteurs des pics apparaissent en figure 1b; des chromatogrammes types d'échantillons biologiques sont fournis en $1 \mathrm{c}$ et $1 \mathrm{~d}$.

Analyses d'acides aminés: Elles ont été effectuées sur les extraits individuels lou après hydrolyse acide : $\mathrm{HCl} 6 \mathrm{~N}, 120^{\circ} \mathrm{C}, 24 \mathrm{~h}$, en présence d'acide thioglycolique $0,1 \%$, en ampoule scellée à une pression inférieure à $25 \mu \mathrm{Hg}$ ). L'analyseur est un Kontron Liquimat III muni d'une résine AS 70 ( $7 \mu \mathrm{m} ; 12 \%$ de réticulation) éluée par 5 tampons Lithium (programme " liquides biologiques »). Le standard de référence est toujours la pNH2 PHE. Un chromatogramme type est montré sur la figure 2.

\section{Résultats.}

Identification de Bg/cTYR. - Le dernier pic (UV $280 \mathrm{~nm}$ ) de la chromatographie préparative est recueilli, cristallisé deux fois dans l'alcool et les cristaux sont comparés au produit de synthèse (Delobel et al., 1984) : point de fusion, co-

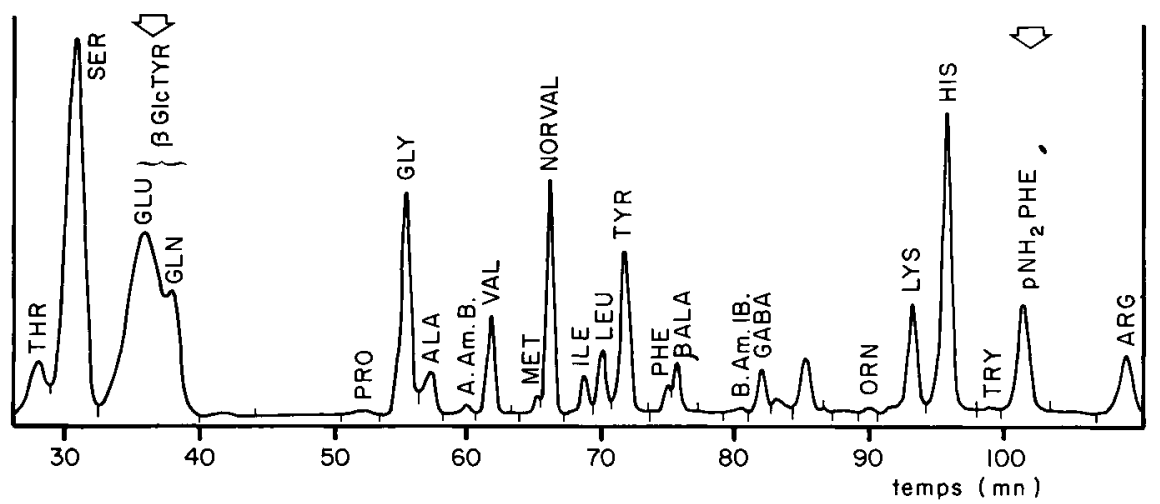

FIG. 2. - Chromatogramme type d'une analyse d'acides aminés libres de larve de stade 6 (temps « $\mathrm{c} »)$. 
chromatographie HPLC et sur l'analyseur d'acides aminés, spectres UV et IR, libération de tyrosine par hydrolyse acide et enzymatique ( $\beta$ glucosidase d'amande) permettent d'identifier le produit obtenu au $\beta$-D-glucopyranosyl-O-L tyrosine. $50 \mathrm{~g}$ de larves ont permis d'obtenir environ $48 \mathrm{mg}$ de glucoside purifié.

Evolution des quantités totales de tyrosine et de son glucoside par animal. Le niveau de tyrosine libre (fig. 3 stades 5 et 6) semble suivre le schéma général périodique d'accumulation entre les mues et d'utilisation lors de celles-ci, déjà décrit en utilisant les dosages microbiologiques (Duchateau-Bosson et al., 1962). Le stade 6, plus détaillé, présente cependant un profil particulier avec une baisse de la tyrosine en début de période d'alimentation (temps a et b) et une augmentation significative après le début du jeûne. BglcTYR est manifestement une forme spécifique du dernier stade larvaire, synthétisée en période d'alimentation active après un délai court mais marqué (environ $24 \mathrm{~h}$ ). Son utilisation est relativement précoce avec deux périodes nettes encadrant un plateau $(+80 \mathrm{~h}$ d'une part et mue nymphale de l'autre). La courbe somme met en outre en évidence une sortie de tyrosine de ce compartiment vers $+80 \mathrm{~h}$ ainsi qu'une légère entrée entre + 102 et $112 \mathrm{~h}$. Il faut noter que ni le bol alimentaire ni les fèces ne contiennent de BglcTYR.

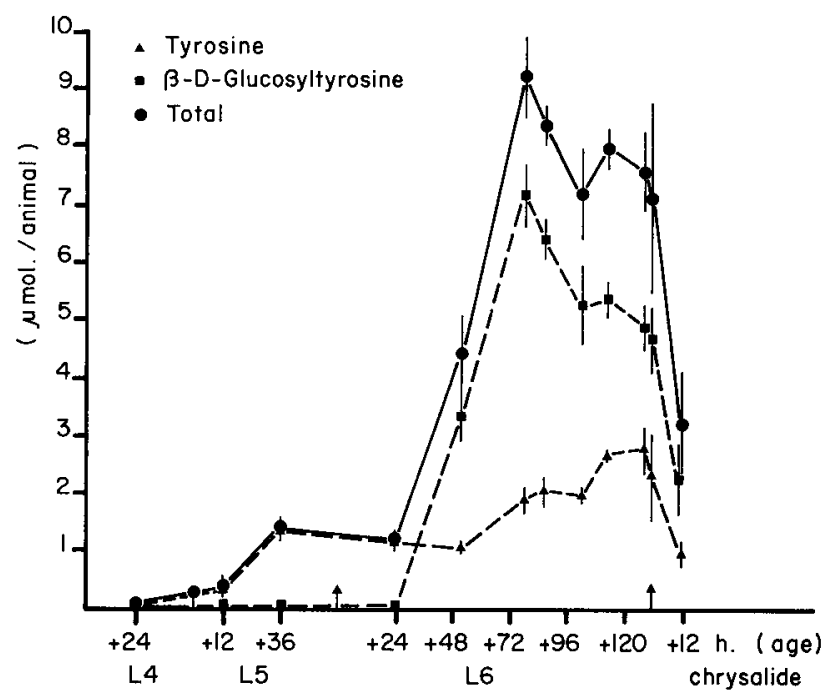

FIG. 3. - Dosage de la tyrosine et de son g/ucoside par individu au cours des 3 derniers stades larvaires de $S$. littoralis (les flèches sur l'axe des abscisses indiquent les mues).

Evolution des concentrations dans l'ensemble des tissus et dans l'hémolymphe. - La concentration de tyrosine libre dans les tissus s'élève régulièrement au cours du $6^{\mathrm{e}}$ stade pour atteindre $9.3 \mathrm{nmol} / \mathrm{mg}$ (tabl. 1), soit une valeur bien audessus de la saturation dans l'eau à $\mathrm{pH}$ neutre $(2.5 \mu \mathrm{mol} / \mathrm{ml})$. La concentration du glucoside, après une rapide et forte hausse, se stabilise grossièrement vers 15 $\mathrm{nmol} / \mathrm{mg}$ jusqu'à la nymphose. La première baisse constatée " par animal " est 
masquée par la perte de poids de la larve (évacuation du contenu intestinal et perte de poids intrinsèque). II est à noter que le tryptophane est affecté par la $5^{\mathbf{e}}$ mue larvaire de façon grossièrement analogue : déplétion à la mue et accumulation après.

\section{TABLEAU 1}

Evolution de la tyrosine, de son g/ucoside et du tryptophane (concentrations) dans l'ensemble des tissus au cours du développement larvaire de $\mathrm{S}$. littoralis

\begin{tabular}{|c|c|c|c|c|c|c|c|}
\hline \multicolumn{4}{|c|}{ Larves } & \multicolumn{4}{|c|}{ Concentration } \\
\hline \multirow{2}{*}{\multicolumn{2}{|c|}{$\begin{array}{c}\text { âge } \\
\text { Temps (heures) } \\
\text { après la mue }\end{array}$}} & \multirow{3}{*}{$\frac{\text { abrév. }}{\omega}$} & \multirow{3}{*}{$\begin{array}{c}\text { poids } \\
\text { moyen (mg) } \\
-\end{array}$} & \multirow[t]{3}{*}{ état } & \multicolumn{3}{|c|}{ (nmoles/mg de poids frais) } \\
\hline & & & & & TYR & $\beta g l c T Y R$ & TRY \\
\hline éclosion & -12 & & & & 1,4 & 0 & 0,085 \\
\hline éclosion & +24 & L1 & $0,11 \pm 0,01$ & actives & $1,3 \pm 0,03$ & 0 & $0,26 \pm 0,03$ \\
\hline $1 / 2$ & +24 & $\mathrm{~L} 2$ & $1,69 \pm 0,04$ & actives & $5,95 \pm 0,10$ & 0 & $0,14 \pm 0,02$ \\
\hline $2 / 3$ & +24 & L3 & $6,92 \pm 0,19$ & actives & $5,2 \pm 0,08$ & 0 & $0,21 \pm 0,01$ \\
\hline $3 / 4$ & +24 & L4 & $25,4 \pm 0,7$ & actives & & 0 & $0,10 \pm 0,01$ \\
\hline $4 / 5$ & +12 & L5a & $88,7 \pm 5,3$ & actives & $3,2 \pm 0,09$ & 0 & $0,14 \pm 0,00$ \\
\hline $4 / 5$ & +36 & L5b & $177 \pm 5$ & actives & $7,3 \pm 1,4$ & 0 & $0,27 \pm 0,07$ \\
\hline $5 / 6$ & +24 & L6a & $295 \pm 43$ & $\begin{array}{l}\text { actives } \\
\text { couleurs vives }\end{array}$ & $3,9 \pm 0,5$ & $0,05 \pm 0,03$ & $30,07 \pm 0,00$ \\
\hline $5 / 6$ & +51 & L6b & $482 \pm 43$ & $\begin{array}{l}\text { actives } \\
\text { alimentation import. }\end{array}$ & $2,3 \pm 0,2$ & $6,8 \pm 0,8$ & $0,05 \pm 0,01$ \\
\hline $5 / 6$ & +78 & L6c & $417 \pm 62$ & $\begin{array}{c} \pm \text { actives } \\
\text { couleurs ternes }\end{array}$ & $4,3 \pm 0,8$ & $16,8 \pm 1,5$ & $0,13 \pm 0,03$ \\
\hline $5 / 6$ & +86 & L6d & $377 \pm 30$ & $\begin{array}{c}\text { inactives } \\
\text { en purge } \\
\text { tête en rétraction }\end{array}$ & $5,2 \pm 0,7$ & $16,9 \pm 1,4$ & $0,23 \pm 0,04$ \\
\hline $5 / 6$ & +102 & L6e & $291 \pm 34$ & $\begin{array}{l}\text { immobiles } \\
\text { ayant filé } \\
\text { rétractées, annelées }\end{array}$ & $6,3 \pm 0,4$ & $15,7 \pm 1,5$ & $0,32 \pm 0,03$ \\
\hline $5 / 6$ & +112 & $L 6 f$ & $279 \pm 29$ & $\begin{array}{l}\text { prénymphes } \\
\text { sensibles }\end{array}$ & $8,25 \pm 0,6$ & $15,6 \pm 2,0$ & $0,32 \pm 0,04$ \\
\hline $5 / 6$ & +128 & L6g & $292 \pm 18$ & $\begin{array}{l}\text { prénymphes } \\
\text { peu sensibles }\end{array}$ & $9,3 \pm 1,2$ & $16,5 \pm 1,0$ & $0,27 \pm 0,05$ \\
\hline nymphose & +0 & $\mathrm{CB}$ & $300 \pm 34$ & $\begin{array}{l}\text { chrysalides blan- } \\
\text { ches }\end{array}$ & $7,5 \pm 1,3$ & $15,5 \pm 0,9$ & $0,27 \pm 0,01$ \\
\hline nymphose & +12 & N & $289 \pm 36$ & chrysalides tannées & $3,1 \pm 0,6$ & $7,6 \pm 1,4$ & $0,32 \pm 0,04$ \\
\hline
\end{tabular}

Les moyennes sont effectuées sur $n=3(\omega-L 4)$ ou $n=5(L 5-N)$ individus (ou groupes) et les temps sont déterminés à $\pm 3 \mathrm{~h}$. 


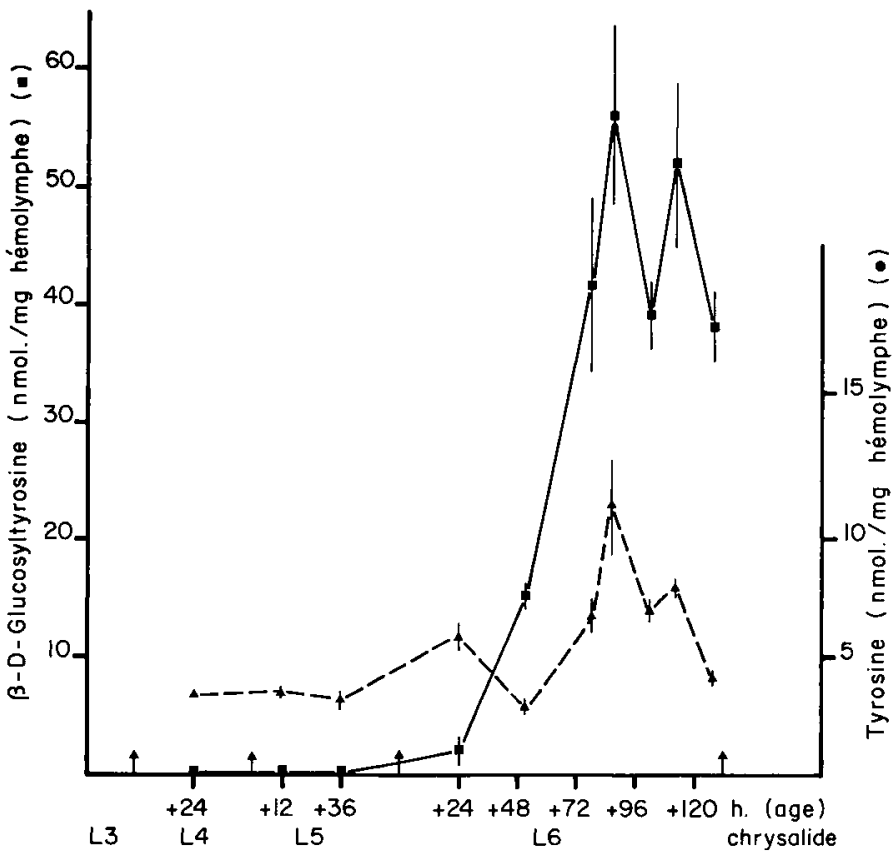

FIG. 4. - Concentrations de TYR et $\beta$ GIc TYR dans l'hémolymphe de S. littoralis au cours des trois derniers stades larvaires.

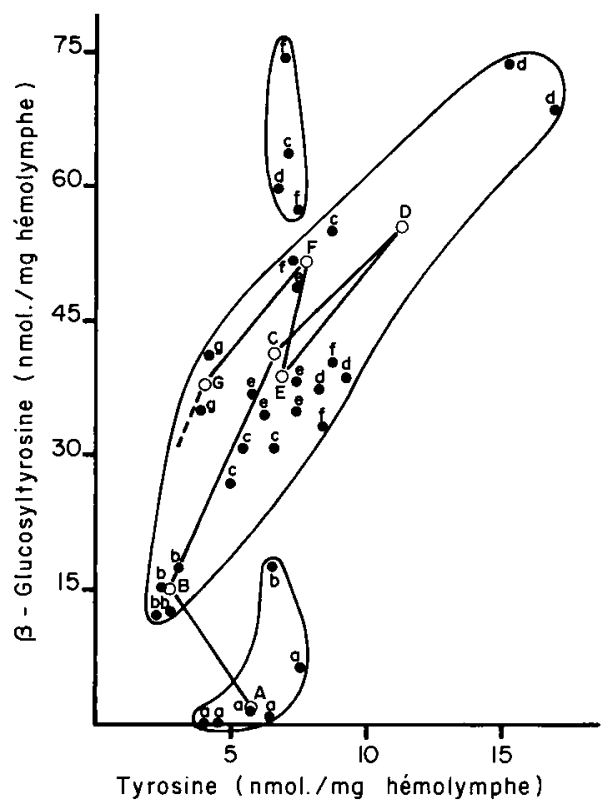

FIG. 5. - Corrélation des évolutions individuelles de TYR et $\beta$ Glc TYR dans l'hémolymphe au cours du dernier stade larvaire (en majuscules, les valeurs moyennes pour chaque temps - voir tabl. 1).

Reproduction, Nutrition, Développement $n^{\circ} 5$ A 1984, -2 . 
Dans l'hémolymphe, les concentrations des deux formes de tyrosine reflètent les variations constatées plus haut avec toutefois une amplitude supérieure (fig. 4). Les interprétations de ces concentrations doivent cependant tenir compte des variations intrinsèques du volume hémolymphatique et des transferts aqueux aux autres tissus. Le synchronisme des variations da la tyrosine et de son glucoside dans l'hémolymphe est illustré par un schéma de corrélation des deux composés sur les données individuelles recueillies (fig. 5). II fait apparaître une

\section{TABLEAU 2}

Evolution des acides aminés libres dans les larves entières de stade 6

Concentration ( $\mu$ moles/animal)

Acide aminé (par groupes)

Temps (age dans L6 - v. tabl. 1)

\begin{tabular}{|c|c|c|c|c|c|c|c|c|c|}
\hline & & a & b & $\mathrm{c}$ & $d$ & $\mathrm{e}$ & $f$ & $\mathrm{~g}$ & $\mathrm{~N}$ \\
\hline $\begin{array}{l}\text { G.A.B.A. } \\
\beta \text { Alanine } \\
\text { Cystathionine } \\
\text { Ornithine } \\
\text { Peptide } 3\left(^{(4)}\right.\end{array}$ & (A) & $\begin{array}{c}4,3 \\
1,45 \\
0,27 \\
0,13 \\
(0,25)\end{array}$ & $\begin{array}{c}15,5 \\
3,52 \\
1,28 \\
1,02 \\
(1,00)\end{array}$ & $\begin{array}{l}3,0 \\
0,65 \\
0 \\
0,03 \\
(0,33)\end{array}$ & $\begin{array}{c}0,19 \\
0,17 \\
0 \\
0,02 \\
(0,31)\end{array}$ & $\begin{array}{l}0 \\
0 \\
0,05 \\
0,02 \\
(0,18)\end{array}$ & $\begin{array}{l}0 \\
0,05 \\
0,04 \\
0,04 \\
0\end{array}$ & $\begin{array}{l}0 \\
0,08 \\
0,05 \\
0,03 \\
0\end{array}$ & $\begin{array}{l}0 \\
0,30 \\
0,08 \\
0,08 \\
0\end{array}$ \\
\hline $\begin{array}{l}\text { Glycine (1) } \\
\text { Thréonine } \\
\text { Valine } \\
\text { Alanine } \\
\text { Leucine }\end{array}$ & (B) & $\begin{array}{l}0,27 \\
0,55 \\
0,46 \\
0,59 \\
0,46\end{array}$ & $\begin{array}{l}4,45 \\
1,68 \\
1,57 \\
1,28 \\
1,15\end{array}$ & $\begin{array}{l}2,66 \\
1,34 \\
1,02 \\
0,60 \\
0,69\end{array}$ & $\begin{array}{l}2,37 \\
0,71 \\
0,85 \\
0,67 \\
0,98\end{array}$ & $\begin{array}{l}0,25 \\
0,51 \\
0,42 \\
0,65 \\
0,25\end{array}$ & $\begin{array}{l}0,45 \\
0,58 \\
0,51 \\
0,53 \\
0,46\end{array}$ & $\begin{array}{l}0,42 \\
0,30 \\
0,31 \\
0,40 \\
0,17\end{array}$ & $\begin{array}{l}0,59 \\
0,42 \\
0,57 \\
0,82 \\
0,44\end{array}$ \\
\hline $\begin{array}{l}\text { Sérine (2) } \\
\text { Glycine (1) } \\
\text { Proline } \\
\text { Isoleucine }\end{array}$ & (C) & $\begin{array}{l}1,59 \\
0,27 \\
0 \\
0,14\end{array}$ & $\begin{array}{l}6,62 \\
4,45 \\
0,75 \\
0,41\end{array}$ & $\begin{array}{l}8,31 \\
2,66 \\
0,92 \\
0,40\end{array}$ & $\begin{array}{l}8,92 \\
2,37 \\
0,76 \\
0,46\end{array}$ & $\begin{array}{l}0,95 \\
0,25 \\
0,30 \\
0,21\end{array}$ & $\begin{array}{l}2,88 \\
0,45 \\
0,42 \\
0,27\end{array}$ & $\begin{array}{l}1,25 \\
0,42 \\
0,20 \\
0,18\end{array}$ & $\begin{array}{l}2,52 \\
0,59 \\
0,22 \\
0,35\end{array}$ \\
\hline $\begin{array}{l}\text { Histidine } \\
\text { Tyrosine } \\
\text { Phénylalanine } \\
\text { Tryptophane (3) }\end{array}$ & (D) & $\begin{array}{l}1,01 \\
0,61 \\
0,16\end{array}$ & $\begin{array}{l}3,15 \\
1,07 \\
0,18\end{array}$ & $\begin{array}{l}3,08 \\
2,07 \\
0,44\end{array}$ & $\begin{array}{l}2,63 \\
1,45 \\
0,40\end{array}$ & $\begin{array}{l}3,58 \\
1,85 \\
0,32\end{array}$ & $\begin{array}{l}4,19 \\
2,36 \\
0,35\end{array}$ & $\begin{array}{l}2,63 \\
1,88 \\
0,20\end{array}$ & $\begin{array}{l}1,66 \\
0,79 \\
0,10\end{array}$ \\
\hline $\begin{array}{l}\text { Arginine } \\
\text { Lysine }\end{array}$ & & $\begin{array}{l}0,66 \\
0,27\end{array}$ & $\begin{array}{l}1,47 \\
0,91\end{array}$ & $\begin{array}{l}1,15 \\
1,12\end{array}$ & $\begin{array}{l}1,07 \\
0,83\end{array}$ & $\begin{array}{l}1,01 \\
0,57\end{array}$ & $\begin{array}{l}1,63 \\
0,92\end{array}$ & $\begin{array}{l}1,11 \\
0,87\end{array}$ & $\begin{array}{l}1,37 \\
1,28\end{array}$ \\
\hline Ac. aspartique & & 0,07 & 0,12 & 0,08 & 0 & 0,19 & 0,08 & 0,08 & 0,12 \\
\hline $\begin{array}{lll}\text { Peptide } & 1 & (4) \\
\text { Peptide } 2 & \left({ }^{4}\right) \\
\text { Peptide } 5 & (4) \\
\text { Peptide } 6 & (4) \\
\text { Peptide } 7 & \left({ }^{4}\right)\end{array}$ & & $\begin{array}{c}(0,52) \\
(0,15) \\
0 \\
0 \\
(0,65)\end{array}$ & $\begin{array}{l}0 \\
(0,12) \\
0 \\
0 \\
(1,00)\end{array}$ & $\begin{array}{c}(0,77) \\
(0,08) \\
0 \\
0 \\
(0,54)\end{array}$ & $\begin{array}{c}(0,44) \\
(0,20) \\
0 \\
0 \\
(0,53)\end{array}$ & $\begin{array}{l}(0,72) \\
(0,39) \\
(0,49) \\
(0,95) \\
(0,39)\end{array}$ & $\begin{array}{l}(0,87) \\
(0,54) \\
(0,54) \\
(1,00) \\
(0,35)\end{array}$ & $\begin{array}{c}(1,00) \\
(0,58) \\
(1,00) \\
0 \\
(0,78)\end{array}$ & $\begin{array}{l}(0,66) \\
(1,00) \\
(0,77) \\
(0,47) \\
(0,38)\end{array}$ \\
\hline
\end{tabular}

(1) GLY présente un profil intermédiaire entre les groupes 2 et 3.

(2) Le pic SER comporte un peptide (Peptide 4) et se trouve donc surestimé, notamment au temps " $c$ » $-20 \%$ environ.

(3) TRY présente le profil de ce groupe mais les valeurs ne proviennent pas de l'analyseur d'acides aminés (v. tabl. 1$)$.

(4) "Peptides " ou composés " ninhydrine + " labiles à l'hydrolyse (v. texte) GLU et GLN ne peuvent être quantifiés directement par suite d'interférence avec $\beta$ glcTYR.

(A).(B).(C).(D) : voir figure 6 . 
bonne correspondance d'ensemble, excepté d'une part les points précédant la mise en place de la synthèse de $\beta$ glcTYR et de l'autre un petit groupe de points issus des périodes précédant les deux phases de dégradation du glucoside. Le parcours moyen peut cependant être considéré comme linéaire (parallélisme des deux courbes de la figure 4 sur le tronçon considéré).

Evolution comparée des acides aminés. - Pour situer les variations de la tyrosine dans un cadre plus général, des échantillons du sixième stade larvaire ont été analysés. Les acides aminés ont été regroupés par comportement analogue au cours de cette période (tabl. 2 ; fig. 6). Les pics non identifiés des chromatogrammes avant hydrolyse, et disparaissant après, ont été considérés comme peptides, numérotés par ordre de sortie, et évalués par rapport à leur concentration maximale notée 1 ; ces pics ne dépassent jamais ceux des acides aminés les moins
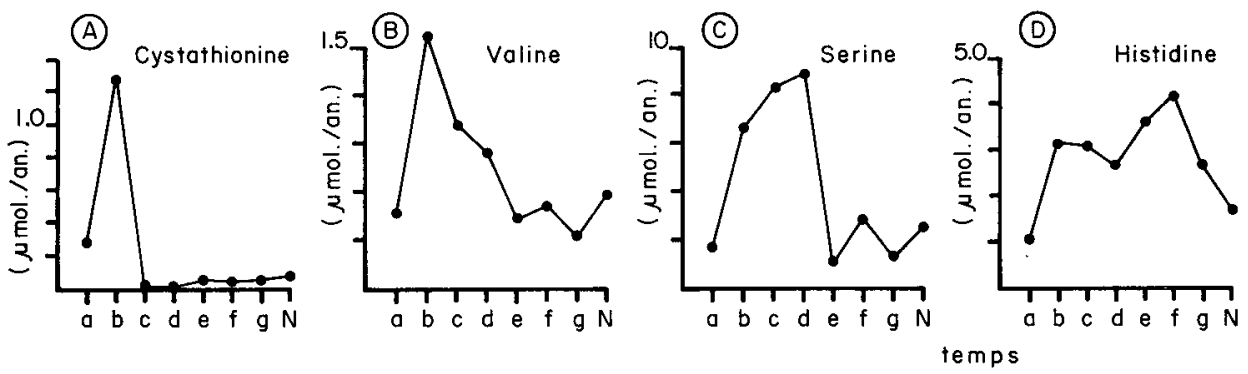

FIG. 6. - Evolution des quantités d'acides aminés par individu au cours du dernier stade larvaire : profils types de chaque groupe.

représentés. La $\beta$ alanine et la phénylalanine sont assez mal séparées et il peut en résulter quelques imprécisions. D'autre part, le peptide 4 ayant le même temps de rétention que la sérine, cette dernière est surévaluée $(20 \%$ minimum en début de stade); les acides aminés peptidiques dosés par différence (après et avant hydrolyse) sont aussi regroupés par analogies de profils (tabl. 3). Les profils types sont cependant moins discriminants : maxima au temps « b » et " $\mathrm{N}$ » (voir tabl. 1), avec une teneur relativement plus élevée au temps « $d$ " pour une majorité d'acides aminés. L'acide glutamique ne peut être évalué directement (pour cause d'interférence avec $\beta$ glcTYR). Il faut enfin noter le peu de $\beta$ alanine peptidique mais l'importance du G.A.B.A. libéré par hydrolyse.

Par ailleurs, si le profil de la tyrosine « peptidique » suit bien celui de la $\beta$ glcTYR dosée par HPLC (tabl. 4), celle-ci reste constamment en excès : or, la comparaison des deux méthodes pour la tyrosine libre montre un bon accord $(\Delta<$ $10 \%$ dans tous les cas). La comparaison des méthodes est faite sur les mêmes échantillons individuels, pour les différents temps; les données du tableau 1 sont des moyennes des mesures HPLC effectuées pour chaque temps. Vu l'existence de peptides contenant de la tyrosine, I'utilisation habituelle de la méthode des différences pour le dosage de $\beta$ glcTYR est ici contre-indiquée (Tabl. 4). 
TABLEAU 3

Evolution des acides aminés peptidiques dans les larves entières de stade 6

\begin{tabular}{|c|c|c|c|c|c|c|c|c|}
\hline \multirow{3}{*}{$\begin{array}{l}\text { Acide aminé } \\
\text { (par groupes) }\end{array}$} & \multicolumn{8}{|c|}{ Concentration ( $\mu$ moles/animal) } \\
\hline & \multicolumn{8}{|c|}{ Temps (age dans L6 - v. tabl. 1) } \\
\hline & a & $\mathrm{b}$ & c & d & e & $f$ & g & $\mathrm{N}$ \\
\hline Ac. aspartique & 0,42 & 1,72 & 0,45 & 0,57 & 0,64 & 0,71 & 0,74 & 1,01 \\
\hline $\begin{array}{l}\text { Proline } \\
\text { Thréonine } \\
\text { Lysine } \\
\text { Alanine } \\
\text { Isoleucine } \\
\text { Leucine }\end{array}$ & $\begin{array}{l}0,28 \\
0,06 \\
0,15 \\
0,09 \\
0,15 \\
0,24 \\
\end{array}$ & $\begin{array}{l}0,75 \\
0,34 \\
0,86 \\
0,40 \\
0,33 \\
0,50 \\
\end{array}$ & $\begin{array}{l}0,20 \\
0 \\
0,13 \\
0 \\
0,14 \\
0,12 \\
\end{array}$ & $\begin{array}{l}0,22 \\
0,12 \\
0,36 \\
0,15 \\
0,21 \\
0,28 \\
\end{array}$ & $\begin{array}{l}0,18 \\
0,09 \\
0,18 \\
0,03 \\
0,16 \\
0,10 \\
\end{array}$ & $\begin{array}{l}0,24 \\
0,01 \\
0 \\
0,05 \\
0,15 \\
0,08 \\
\end{array}$ & $\begin{array}{l}0,18 \\
0,06 \\
0,21 \\
0,14 \\
0,13 \\
0,09 \\
\end{array}$ & $\begin{array}{l}0,47 \\
0,24 \\
0,47 \\
0,26 \\
0,26 \\
0,34 \\
\end{array}$ \\
\hline $\begin{array}{l}\text { Valine } \\
\text { Glycine }\end{array}$ & $\begin{array}{l}0,09 \\
2,25\end{array}$ & $\begin{array}{l}0,29 \\
4,90\end{array}$ & $\begin{array}{l}0,14 \\
3,21\end{array}$ & $\begin{array}{l}0,31 \\
5,67\end{array}$ & $\begin{array}{l}0,19 \\
3,50\end{array}$ & $\begin{array}{l}0,19 \\
3,70\end{array}$ & $\begin{array}{l}0,20 \\
2,90\end{array}$ & $\begin{array}{l}0,25 \\
4,98\end{array}$ \\
\hline $\begin{array}{l}\text { Arginine } \\
\text { Histidine }\end{array}$ & $\begin{array}{l}0,23 \\
0,10\end{array}$ & $\begin{array}{l}0,36 \\
\mathbf{0 , 4 6}\end{array}$ & $\begin{array}{l}0,42 \\
0,18\end{array}$ & $\begin{array}{l}0,27 \\
\mathbf{0 , 7 2}\end{array}$ & $\begin{array}{l}0.52 \\
0.84\end{array}$ & $\begin{array}{l}0,38 \\
0,07\end{array}$ & $\begin{array}{l}0,56 \\
0,14\end{array}$ & $\begin{array}{l}0,65 \\
0,34\end{array}$ \\
\hline Tyrosine (1) & 0,09 & 4,28 & 9,30 & 9,03 & 8,52 & 7,62 & 6,54 & 1,08 \\
\hline Phénylalanine & 0,15 & 0,24 & 1,05 & 0,27 & 0,13 & 0,07 & 0,05 & 0,11 \\
\hline $\begin{array}{l}\text { G.A.B.A. } \\
\beta \text { Alanine }\end{array}$ & $\begin{array}{l}1,79 \\
0,29\end{array}$ & $\begin{array}{l}2,52 \\
0.11\end{array}$ & $\begin{array}{l}0,81 \\
0\end{array}$ & $\begin{array}{l}0,06 \\
0\end{array}$ & $\begin{array}{l}0,17 \\
0\end{array}$ & $\begin{array}{l}0,21 \\
0\end{array}$ & $\begin{array}{l}0,18 \\
0\end{array}$ & $\begin{array}{l}0 \\
0\end{array}$ \\
\hline Ac. Glutamique (2) & 2,31 & 7,07 & 3,87 & 4,41 & 3,21 & 3,68 & 3,02 & 2,94 \\
\hline
\end{tabular}

(1) Les valeurs de ce tableau sont obtenues par différence - après et avant hydrolyse - . La tyrosine $"$ peptidique » comprend donc $\beta$ glcTYR (v. tabl. 4).

(2) Somme des acides aminés libres et peptidiques (GLU et GLN).

\section{TABLEAU 4}

Comparaison du dosage de Bg/cTYR par HPLC et comme tyrosine liée (analyseur)

\begin{tabular}{|c|c|c|c|c|c|c|c|c|}
\hline & \multicolumn{8}{|c|}{ Concentration (nmoles/mg poids frais) } \\
\hline & \multicolumn{8}{|c|}{ Temps (age dans L6 - v. tabl. 1) } \\
\hline & a & $b$ & c & d & e & $f$ & g & $\mathrm{N}$ \\
\hline $\begin{array}{c}\text { A ßglucosyl Tyrosine } \\
\text { (HPLC) }\end{array}$ & 0 & 8,3 & 18,9 & 20,9 & 20,3 & 16,4 & 18,4 & 3,1 \\
\hline $\begin{array}{l}\text { B Tyrosine "peptidique " } \\
\text { (analyseur) }\end{array}$ & 0,5 & 8,7 & 20,5 & 23,5 & 29,5 & 21,2 & 25,2 & 3,4 \\
\hline$\Delta \%(B-A / A \times 100)$ & - & $+4,8$ & $+8,5$ & $+12,4$ & $+45,3$ & $+29,3$ & $+40,0$ & $+9,7$ \\
\hline
\end{tabular}




\section{Discussion.}

Chez les lépidoptères l'utilisation de $\beta$ glucosyl-tyrosine pour stocker la tyrosine apparaît fortement liée à la métamorphose. Notre travail confirme les résultats récents limitant la présence du glucoside au seul dernier stade larvaire (Ahmed et al., 1983a) et à la forme nymphale (lsobe et al., 1981). Cette forme de mobilisation rapide, possédant une solubilité molaire dans l'eau 25 fois supérieure à celle de la tyrosine libre, partage le rôle de "réservoir aromatique " pour la métamorphose avec une protéine spécifique génériquement nommée arylphorine par Telfer et al. (1983). Cette protéine s'accumule parallèlement à $\beta$ glcTYR dans le dernier stade larvaire, à un taux molaire maximal en tyrosine équivalent : 31,6 nmoles de tyrosine par $\mathrm{mg}$ d'hémolymphe pour la manducine et $29,2 \mathrm{nmole} / \mathrm{mg}$ pour $\beta$ glcTYR, chez $M$. sexta (d'après les données respectives de $\mathrm{S}$. J. Kramer et al., 1980 et Ahmed et al., 1983a). Signalons au passage la plus grande teneur maximale en glucoside chez $S$. littoralis $(55 \mathrm{nmoles} / \mathrm{mg}$ d'hémolymphe). Les arylphorines se voient attribuer des rôles allant d'un stockage ultérieur de tyrosine, au niveau du corps gras nymphal (S. J. Kramer et al., 1980), à une incorporation dans la cuticule nymphale puis adulte (Scheller, 1982).

La synthèse très rapide de $\beta$ glcTYR pendant la période d'alimentation intense met l'accent sur un lien possible entre les deux phénomènes. La quantité moyenne de tyrosine ingérée journellement par $S$. littoralis est évaluée par des mesures de consommation à environ $4,8 \mu$ moles/animal, alors que la synthèse de $\beta$ glcTYR en utilise environ 3,2 $\mu$ moles/animal et par jour. Par ailleurs, l'utilisation digestive apparente de la tyrosine a été estimée à $80 \%$ (alors que les autres acides aminés, phénylalanine comprise, montrent une U.D. apparente variant de 55 à $70 \%$; seules la méthionine et l'arginine montrent aussi des valeurs supérieures à $70 \%)$. Dans ces conditions, la tyrosine disponible $(3,85 \mu$ moles/animal) couvre juste les besoins de synthèse du glucoside, et il semble logique de postuler l'existence d'un stockage larvaire précoce pour répondre à la demande globale de tyrosine.

La dégradation de $\beta$ glcTYR présente deux phases que l'on peut chronologiquement mettre en relation avec les deux pics d'ecdystéroides signalés pendant cette période, par exemple chez une noctuelle voisine $M$. brassicae (Agui et Hiruma, 1982). Les derniers résultats de Ahmed et al. (1983b) sont en accord avec cette hypothèse et semblent désigner le corps gras comme principal foyer d'activité de l'hydrolase correspondante, probablement une $\beta$ glucosidase. Si le deve-

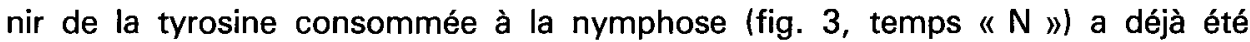
signalé, la diminution précoce du niveau de tyrosine (fig. 3 et 4 , temps « $d$ ") est d'interprétation moins évidente. Elle peut être liée soit à une synthèse protéique - arylphorine -, soit à une séquestration et utilisation par certains tissus, par exemple pour l'élaboration de la soie (chez B. mori ; Duchateau-Bosson et al., 1962) ou de la case nymphale.

Par ailleurs, si l'on estime le volume d'hémolymphe d'une larve par majoration de $20 \%$ du volume d'une ponction totale moyenne (environ $90 \mu \mathrm{l}$ au temps " $\mathrm{d} \|)$, on s'aperçoit que la quantité de $\beta$ glcTYR contenue dans l'hémolymphe $(6,05 \mu$ móles) correspond à peu près à celle contenue dans une larve entière $(6,40$ 
$\mu$ moles). L'hémolymphe paraît donc bien être le compartiment presque unique de stockage du glucoside; de plus la corrélation assez marquée illustrée en figure 5 laisse prévoir un couplage étroit des métabolismes de la tyrosine et de son glucoside au sein de ce compartiment.

Il est par ailleurs important de situer le comportement de la tyrosine par rapport à celui des autres acides aminés. On remarque qu'elle se distingue bien des deux groupes d'acides aminés dont les pics d'accumulation correspondent à la période d'alimentation (groupe GLY, THR, VAL, ...) et au métabolisme qui y est associé (G.A.B.A., $\beta$ ALA, cystathionine) ; il en est de même vis à vis du groupe contenant la sérine qui pourrait être lié à certains métabolismes particuliers (filage de la soie, changement du catabolisme azoté-Buckner et Caldwell, 1980). Finalement, parmi les acides aminés à profil d'accumulation de type tyrosine, on trouve la phénylalanine, qui peut elle aussi être incorporée à la cuticule par hydroxylation en tyrosine, et le tryptophane qui ne paraît cependant pas être utilisé à la mue nymphale de la même façon qu'aux mues larvaires (pour les pigments oculaires et tégumentaires). Le cas de l'histidine dont la teneur augmente considérablement, peut s'interpréter comme une accumulation d'un acide aminé à très faible catabolisme (Nakamura et al., 1977) et/ou comme un résidu basique, donneur de groupements NH2, incorporé dans les protéines cuticulaires (Richards, 1978).

La plupart des acides aminés peptidiques montrent une concentration maximale au deuxième jour, ainsi qu'à la nymphose. Quantitativement, on retrouve les principaux acides aminés signalés dans les peptides de diptères (GLY, GLX, ASX), lesquels sont considérés comme des formes de stockage non spécifiques des acides aminés hémolymphatiques régulés par des peptidases (Collet, 1976). Le G.A.B.A. peptidique est probablement dû à la décarboxylation d'acide glutamique peptidique, et il faut noter, en plus de $\beta$ glcTYR, la présence en quantité non négligeable de tyrosine et de phénylalanine peptidiques (tabl. 3 et 4 ). Par ailleurs, on remarque les acides aminés basiques arginine et histidine dans un groupe non lié à l'alimentation, peut-être analogues à ceux intervenant dans les dipeptides de diptères riches en histidine et en lysine (Bodnaryk, 1978), et dont la fonction reste inconnue.

La compartimentation de la tyrosine au cours du développement larvaire des lepidoptères présente des particularités importantes vis-à-vis de celles des autres acides aminés. Sa caractérisation plus complète permettrait de mieux comprendre la complexité des mécanismes physiologiques et biochimiques mis en œuvre ; l'étude de l'arylphorine de $S$. littoralis est en cours dans ce but.

Reçu en décembre 1983. Accepté en avril 1984.

\section{Références}

AGUI N., HIRUMA K., 1982. Ecdysteroïd titer and its critical period during larval and pupal ecdysis in the cabbage armyworm Mamestra brassicae L. Appl. entomol. Zool., 17, 144-146.

AHMED R. F., HOPKINS T. L., KRAMER K. J., 1983a. Tyrosine and tyrosine glucoside titers in whole animals and tissues during development of the tobacco hornworm Manduca sexta. Insect Biochem., 13, 369-374. 
AHMED R. F., HOPKINS T. L., KRAMER K. J., 1983b. Tyrosine glucoside hydrolase activity in tissues of Manduca sexta L. : effect of $20 \mathrm{OH}$ ecdysone. Insect Biochem., 13, 641-645.

ANDERSEN S. O., 1971. Phenolic compounds isolated from insect hard cuticle and their relationships to the sclerotization process. Insect Biochem., 1, 157-170.

BODNARYK R. P., 1978. Structure and function of insect peptides. Adv. Insect Physiol., 13, 69-132.

BUCKNER J. S., CALDWELL J. M. 1980. Uric acid levels during last larval instar of Manduca sexta, an abrupt transition from excretion to storage in fat body. J. Insect Physiol., 26. 27-32.

COLLET J. R., 1976. - Peptidase mediated storage of aminoacids in small peptides. Insect Biochem., 6, 179-185.

DELOBEL B., ANKER D., GRENIER S., 1984. Synthèse chimique de la $\beta$-D-glucopyranosyl-Otyrosine et utilisation par un insecte endoparasite. Repr. Nutr. Dévelop. (soumis).

DUCHATEAU-BOSSON G., JEUNIAUX C., FLORKIN M., 1962. Contribution à la biochimie du ver à soie. Variations de la concentration de la tyrosine de l'hémolymphe au cours du développement de Bombyx mori L. Arch. int. Physiol. Bioch., 70, 287-291.

ISHIZAKI Y., UMEBACHI Y., 1980. The presence of tyrosine glucoside in the hemolymph of lepidopteran insects. Sci. Rep. of Kanazawa, 25, 43-52.

ISOBE M., KONDO N., IMAI K., YAMASHITA O., GOTO T., 1981. Glucosyl tyrosine in silkworm hemolymph as a transient metabolite of insects. Agric. biol. Chem., 45, 687-692.

KARLSON P., SEKERIS C. E., 1962. $\mathrm{N}$ acetyl dopamine as sclerotizing agent of the insect cuticle. Nature, London, 195, 183-184.

KRAMER K. J., HOPKINS T. L., AHMED R. F., MUELLER D., LOOKHART G., 1980. Tyrosine metabolism for cuticle tanning in the tobacco hornworm Manduca sexta and other Lepidoptera : identification of $\beta$-D-glucopyranosyl-O-L-tyrosine and other metabolites. Arch. Biochem. Biophys., 205, 146-155.

KRAMER S. J., MUNDALL E. C., LAW J. H., 1980. Purification and properties of manducin, an aminoacid storage protein of the haemolymph of larval and pupal Manduca sexta. Insect Biochem., 10, 279-288.

LU PW., KRAMER K. J., SEIB P. A., MUELLER D. D., AHMED R., HOPKINS T. L., 1982. $\beta$-D-glucopyranosyl-O-L tyrosine : synthesis, properties and titer during insect development. Insect Biochem., 12, 377-381.

McDERMID H., LOCKE M., 1983. Tyrosine storage vacuoles in insect fat body. Tissue Cell, 15, 137-158.

NAKAMURA K., INOKUCHI T., 1977. Free aminoacids in the meconium and distribution of histidine in the pupa of silkworn. B. mori. J. Seric. Sci. Jap., 46, 125-129.

POITOUT S., BUES R., LE RUMEUR C., 1972. Elevage, sur milieu artificiel simple, de deux noctuelles parasites du coton : Earias insulana et Spodoptera littoralis. Ent. exp. appl., 15. 341-350.

RICHARDS G., 1978. The chemistry of insect cuticle, 203-232. In ROCKSTEIN M., Biochemistry of insects. Acad. Press.

SCHELLER K., 1982. The larval serum proteins of insects. Eur. J. Cell Biol., 28, 281-282.

SCHUSTER R., 1980. Determination of free aminoacids by high performance liquid chromatography. Anal Chem., 52, 617.

SUGUMARAN M., LIPKE H., 1982. Crosslink precursors of the dipteran puparium. Proc. nat. Acad. Sci. U.S.A., 79, $2480-2484$.

TELFER W. H., KEIM P. S., LAW J. H., 1983. Arylphorin a new protein from Hyalophora cecropia : comparisons with calliphorin and manducin. Insect Biochem., 13, 601-613. 